\title{
Timing is everything
}

\author{
Ami E. Iskandrian, MD, MASNC ${ }^{a}$ \\ a University of Alabama at Birmingham, Birmingham
}

Received Dec 8, 2020; accepted Dec 8, 2020

doi: $10.1007 /$ s12350-020-02490-w See related article, doi:https://doi.org/10.
1007/s1 2350-021-02553-6.

It is unusual that the Journal publishes an editorial on a case report (referred to as Images That Teach) but I thought the case by Aikawa et al in this issue of the Journal deserves one because it provides so many teaching points that are very useful to the health providers in general and to imagers (of any imaging modality) in particular. ${ }^{1}$ The authors present in exquisite details and high-quality images the findings of a patient with suspected CAD.

The patient had ECG, 2-dimensional echocardiogram, CT coronary angiography (CTCA), and dipyridamole stress/rest myocardial perfusion imaging (MPI) using Tc-99m Tetrofosmin. The patient had mild atherosclerosis by CTCA but developed ST elevation during the stress test; the stress perfusion pattern, however, was normal though the wall motion in the distribution of the left anterior descending artery (LAD) which had the mild atherosclerosis by CTCA was abnormal. The rest images on the other hand, showed abnormal perfusion in the distribution of LAD but normal wall motion. Before the reader invokes falsepositive or false-negative concepts or improper interpretation, few of the details may help calm the nerves.

The patient had no chest pain or ECG changes during the dipyridamole infusion, both appeared 8 minutes later. The pain and ST elevations (figure 1 of their paper) subsided after nitroglycerin. The pain recurred again but without ST elevation (only T-wave inversions) before the rest images were obtained. The authors raise the possibility of reverse redistribution pattern on the perfusion images.

Reprint requests: Ami E. Iskandrian, MD, MASNC, University of Alabama at Birmingham, Birmingham; aiskand@uab.edu J Nucl Cardiol 2021;28:2465-6.

1071-3581/\$34.00

Copyright (C) 2021 American Society of Nuclear Cardiology.
I will address the following issues: reverse redistribution, stunning and ST shifts with vasodilator stress testing, and offer my explanation to the findings in this case.

Reverse redistribution (worsening rather than improvement of perfusion pattern on the delayed images compared to initial images) was first described with thallium-201 as a marker of successful thrombolysis in patients with acute myocardial infarction (MI). The mechanism was thought to be due to faster washout from salvaged myocardium compared to the remaining myocardium. We all have seen examples of this phenomenon, but we also have seen it in patients without acute MI or without CAD.

The whole interest in washout kinetics disappeared when Tc-labeled radiotracers replace thallium-201 but the reverse redistribution aspect never died completely even though with TC tracers, 2 separate injections are made (stress and rest) and hence the whole concept of differential washout or clearance simply does not exist. I have never mentioned reverse redistribution in any of my reports using Tc tracers and I suggest imagers should do same. It is not that we do not see it but in my judgment has no relevance or alternative explanations.

Post-stress stunning is manifested as transient regional (wall motion/thickening abnormality) or global (decrease in LV ejection fraction) dysfunction after stress imaging but not after rest imaging. it is a real phenomenon and the earlier the images are obtained after stress, as when using positron emission tomography for MPI, the more likely it is detected. It lasts for a variable period of time but very likely longer with more severe (transmural ischemia) or repetitive episodes than after milder (sub-endocardial) ischemia.

ST shifts (usually ST depression) can occur with vasodilator imaging but ST elevation is much less common. Unlike ST depression, ST elevation localized the culprit vessel, in this case the LAD. ST elevation could be due to severe epicardial stenosis (with superimposed spasm) or mild or no stenosis and spasm is the only explanation. We have seen examples of both. It is uncertain whether ST elevation could be produced by 
microvascular dysfunction alone. Parenthetically, perfusion defects due to spasm are generally of severe degree, as the myocardial blood flow (MBF) is below baseline in the culprit zone unlike the typical perfusion defects which reflect unequal increases in MBF between diseased and normal vessels.

What about the patient in this report? I suspect, the spasm occurred after the tracer injection and hence the perfusion pattern was normal, but the post-stress stunning was evident by the regional dysfunction.

He subsequently developed several episodes of less severe spasm as denoted by lack of ST elevation and conceivably some of these episodes were asymptomatic and by luck the second dose of tracer was injected during one of these episodes (and hence the perfusion abnormality) but since the ischemia was mild, the subsequent stunning was short-lived and there was no regional dysfunction at rest.
The management of this patient is not that easy and sudden death has been reported in patients with coronary spasm but long-term treatment with long acting nitrates, calcium channel blockers, and lipid lowering agents are needed and non-imaging stress testing and Holter monitoring are needed to assess response.

Finally, do we need to invoke revere-redistribution? No!

\section{Reference}

1. Aikawa T, Funayama N, Sunaga D, Kayanuma K, Oyama-Manabe N, Daisuke Hotta D. Reverse redistribution-like change on dipyridamole-stress $99 \mathrm{mTc}$-tetrofosmin imaging in a patient with angiographically mild coronary artery stenosis. J Nucl Cardiol 2021.

Publisher's Note Springer Nature remains neutral with regard to jurisdictional claims in published maps and institutional affiliations. 